\title{
Using Evidential Occupancy Grid for Vehicle Trajectory Planning Under Uncertainty with Tentacles
}

\author{
Hafida Mouhagir ${ }^{1,2}$, Véronique Cherfaoui ${ }^{1}$, Reine Talj ${ }^{1}$, François Aioun ${ }^{2}$, Franck Guillemard ${ }^{2}$
}

\begin{abstract}
The uncertainty in environment perception is one of the challenges that we face in trajectory planning. For autonomous vehicle to be efficient, they need to be able to deal with this kind of uncertainty. In this work, we combine two existing frameworks: the Belief Functions to build evidential occupancy grid and clothoid tentacles for trajectory planning. First, we use evidential grids to represent the environment and the uncertainties which arise from ignorance and errors during the perception process. Secondly, we generate a set of clothoid tentacles in the egocentered reference frame related to the ego-vehicle, those tentacles represent possible local trajectories. Thirdly, we modify the evidential grid in order to take into consideration some traffic rules such as safety distance between vehicles. Then to choose the best tentacle to execute, we use reward system of a Markov Decision Process-like model to evaluate generated tentacles regarding several criteria including uncertainty represented by the evidential grid. Real and simulated data were used to validate the planning algorithm with evidential grids.
\end{abstract}

\section{INTRODUCTION}

Autonomous driving requires decision making in dynamic and uncertain environments. The uncertainties come from: imperfect knowledge of the vehicle model noisy sensor data, occlusions in the perception system, and poor predictability due to the inability of measuring other driver's intentions.

To solve environment predictability, Partially Observable Markov Decision Process (POMDP) [8] is a method which allows to find an optimal action given the uncertainty of the perception system and/or future behavior. It provides near optimum solutions for decision making with a variable number of traffic participants and with unknown maneuver intentions. This approach expects that the ego-vehicle will continuously gather information about its surrounding and incorporates them in the decision making.

Brechtel et al. [3] use Continuous POMDP in decision making to address both problems of noisy sensor measurement and the environment occlusion in intersection scenarios. The authors of [19] presented a QMDP-based approach (QMDP is a hybrid between MDP and POMDP, this algorithm generalizes the MDP-optimal value function defined over states, into a POMDP-style value functions over beliefs) for singlelane behaviors. They show that considering uncertainty in the behavior of the leading vehicle as well as limitations of the perception improves robustness. However, they used a state space that is tailored for single-lane driving.

The authors are with ${ }^{1}$ Sorbonne universités, Université de Technologie de Compiègne (UTC), CNRS Heudiasyc UMR 7253, ${ }^{2} P S A$ Groupe, Direction scientifique, Centre technique de Vélizy, France. Email: \{hafida.mouhagir, reine.talj, veronique.cherfaoui\}@hds.utc.fr, \{franck.guillemard, françois.aioun\}@mpsa.com
In this work, we focus on the grid-based approach to model the environment, including the obstacle information. The reason behind this choice instead of object level approach for example is that we are looking to have a planning method that works with as few sensors as possible.

The occupancy grids are constructed by interpreting the sensor information into the grid cell values. When interpreting the sensor data into occupancy information, uncertainty inevitably arises from ignorance and errors. Ignorance is due to the perception of new areas or to occlusions and errors come from noisy measurements and imprecise pose estimation. In the literature, the Bayesian framework is the most popular method to tackle this problem by representing the uncertainties by means of probability, then the update step adopts the Bayesian Theorem to fuse new information. In [5], the authors present a method to estimate the probability of collision with uncertainty in position, shape and velocity of the obstacles. They used Bayesian Occupancy Filter (BOF) witch is a dynamic occupancy grid where an estimation of velocity is stored as well as the probability of occupation. However, occlusions and free space have the same low probability of occupation. This problem can be solved using the theory of belief function.

First introduced by [4] and formalized by [16], the framework of belief function has a growing number of applications in artificial intelligence, information fusion, classification, reliability and risk analysis, etc. In [13], the authors used this framework to build evidential occupancy grid that provides the ego-vehicle with additional information about its environment. They detect moving objects by analyzing conflicting information.

In this work, we use evidential grids elaborated thanks to a lidar range scanner to model the uncertainties of the environment. Once the information on the surrounding environment is provided by the grid, the next step is to interpret this information to plan a trajectory using clothoid tentacles. This trajectory planning approach [7] considers the current dynamical state of the vehicle and makes a smooth variations in the vehicle dynamic variables.

The contribution presented in this paper is the combination of two existing frameworks: The Belief Functions to build occupancy grid and clothoid tentacles for trajectory planning. We modify the evidential grids to take into account the safety distances between the ego-vehicle and obstacles. To choose the best tentacle to execute, we use reward system of a Markov Decision Process-like model to evaluate generated tentacles regarding several criteria including uncertainty represented by the evidential grid. Real and simulated data were used to validate the approach. 
In Section II, we present the construction of the evidential grids for trajectory planning. In Section III, the trajectory planning algorithm and the reward system of the $M D P$ like model are explained. First results based on real and simulated data are discussed in Section IV followed by a conclusion of the paper including an outlook.

\section{EVIDENTIAL OCCUPANCY GRID}

The occupancy grids are used as an environment model, if the grid cells are filled with obstacle information in the form of evidence (mass or belief values for instance II-A), we call this kind of grids "Evidential occupancy grids".

\section{A. Evidential framework}

The theory of belief functions, also known as Dempster-Shafer theory (DST), was proposed by Dempster [4], and developed, among others, by Shafer [16] and Smets [18].

Let $w$ be an unknown quantity with possible values in a finite domain $\Omega$, called the frame of discernment. A piece of evidence about $w$ may be represented by a mass function $m$ on $\Omega$, defined as a function $2^{\Omega} \rightarrow[0,1]$, such that $m(\emptyset)=0$ and $\sum_{A \subset \Omega} m(A)=1$.

In the theory of Dempster-Shafer, a frame of discernment $\Omega$ is defined to model a specific problem. In the occupancy grid framework, the frame of discernment is defined as: $\Omega=$ $\{F, O\}$, referred as the states (free or occupied) of each cell. The power set is defined as $2^{|\Omega|}=\{\emptyset, F, O, \Omega\}$, with $|\Omega|$ is the cardinality of the set.

For quantitatively supporting the cell states, a mass function (also referred as Basic Belief Assignment $B B A$ ) is calculated and provides four beliefs $[m(\emptyset) m(F) m(O) m(\Omega)]$, where $m(A)$ represents respectively the quantity of evidence that the space is Conflict, Free, Occupied, and Unknown.

\section{Combination rules}

There is a large panel of combination rules to fuse BBAs (or beliefs or mass functions) coming from independent sources. Usually, the BBAs should be defined in the same frame of discernment. We describe the two most used ones in data fusion:

- The conjunctive rule proposed by Smets, is used to combine two BBAs provided by reliable and distinct information sources [17]. The resulting BBA, denoted $m_{1 \cap 2}$, is defined by:

$$
m_{1 \cap 2}(A)=\sum_{B \cap C=A} m_{1}(B) m_{2}(C), \quad \forall A \subseteq \Omega
$$

The mass assigned to the empty set $m_{1 \cap 2}(\emptyset)$ quantifies the degree of disagreement between the two combined sources.

- The Dempster rule, based on the orthogonal sum, is a normalized version of the conjunctive rule where the mass of the empty set (mass on conflict) must be reallocated over all focal elements in the case where $m_{1 \cap 2}(\emptyset) \neq 0$ thanks to a normalization factor, denoted $K$ [16]. This rule, assuming pieces of evidence combined to be reliable and distinct, is defined as follows:

$$
m_{1 \oplus 2}(A)=K m_{1 \cap 2}(A), \quad \forall A \subseteq \Omega
$$

$$
\text { and } m_{1} \oplus m_{2}(\emptyset)=0 \text { where } K=\left(1-m_{1 \cap 2}(\emptyset)\right)^{-1}
$$

\section{B. Evidential occupancy grid}

In this section, we present the perception grids used in our approach. The construction of these grids is based on data coming from a range sensor that provides information about the occupancy/free of the cells.

The first step as described in [12], consists on computing the PerceptionGrid from successive Lidar scans. For every sensor measurement, a ScanGrid is built with sensor model that translates the sensor information into an ego-centered grid. The BBA assignment respects the least commitment principle: the cells containing a Lidar point are occupied, the cells between the sensor and the occupied cells are free and the other are unknown. The value of masses depends of the resolution of the grids and sensor performances.

The successive ScanGrids are fused in a unique resulting PerceptionGrid. To combine the new ScanGrid with the current PerceptionGrid, two operations are carried out: first, a transformation (rotation and translation) computed with the vehicle displacement is applied to the ScanGrid, and then all masses of the PerceptionGrid are discounted to give less importance to the past (Fig. 1). The fusion rule is based on the conjunctive rule that can provide conflicting mass given information about moving cells.

After PerceptionGrid processing, each cell has a mass function with four beliefs on the state of the cell $[m(\emptyset) m(F) m(O) m(\Omega)]$. Let consider a concrete case to illustrate these concepts, $[m(\emptyset) m(F) m(O) m(\Omega)]=\left[\begin{array}{llll}0 & 0 & 0.7 & 0.3\end{array}\right]$ indicates an Occupied cell with 0.7 as a belief, the rest of the mass is in Unknown. $[m(\emptyset) m(F) m(O) m(\Omega)]=\left[\begin{array}{llll}0 & 0.6 & 0 & 0.4\end{array}\right]$ shows we have belief 0.6 in Free state, the rest of mass is in Unknown.

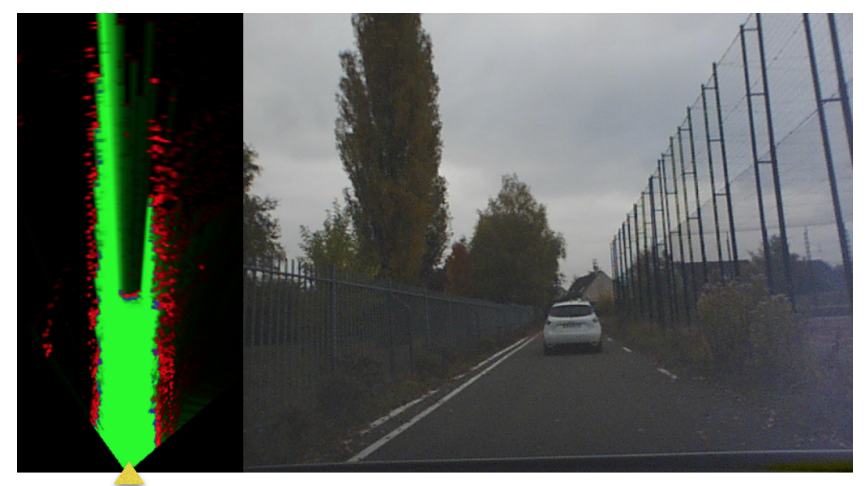

Figure 1: Example of an occupancy grid with its corresponding scene. The yellow triangle represents the position of the Lidar sensor, the green color in the occupancy grid shows the free space, the red shows the occupied space, while the blue represents conflicting cells (before normalization) and the black represents unexplored cells. The color intensity reflects the certainty degree.

Once the perception grid is created, we add both the map information with road limits witch make it possible to distinguish between free navigable or non navigable space and dynamic obstacles velocity obtained using car-to-car communication. With this new integrated information a new grid is obtained. 


\section{Evidential planning grid}

The evidential occupancy grids provide information about the occupation of the environment, this information are used to plan a trajectory to avoid collisions. However, the autonomous vehicles must be capable of avoiding collision, lane keeping and carrying out an overtaking maneuver while keeping safety distances. Therefore, we propose to modify the grid by adding information about the edges of the road and by expanding the dynamic obstacles to include safety distances. The resulting grid is what we call PlanGrid for planning grid.

In Fig. 2- b), an example of enhancement of the map with road limit is given. The position of the vehicle and the information of the map was used to add a simple mask to the evidential grid which integrates the edges of the road by modifying the BBA of the cells out of the road surface. More elaborated methods of road segmentation exist. In the literature, the authors of [2] propose a learning method for road scene segmentation from a single image; the authors of [20] use Lidar as a sensor and prior maps and accurate pose estimation.

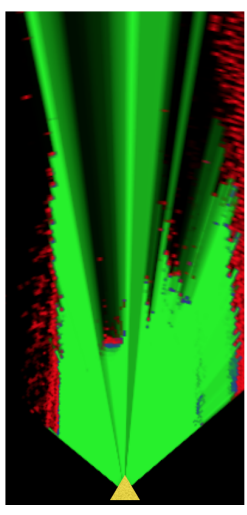

(a)

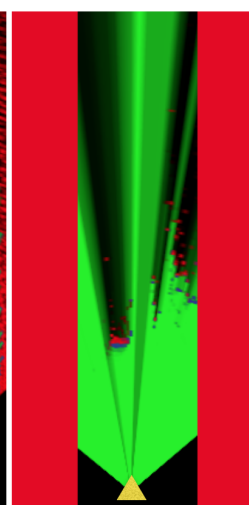

(b)

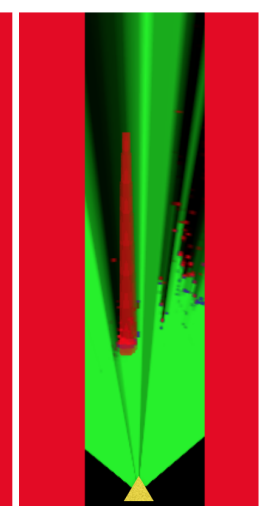

(c)
Figure 2: The PlanGrid (b) represents PerceptionGrid (a) with the road's edges information. The PlanGrid (c) presents a longitudinal obstacle widening.

In order to respect safety distances, the last step for building the PlanGrid is the longitudinal expansion of dynamic obstacles in the occupancy grid (Fig. 2- $c$ and Fig. 3). We propose to extend our previous work on the safety distance in binary grids [14] considering both : the uncertainties modeled by mass function and the safety distance $S_{\text {safe }}$ calculated with formula proposed by [1]:

$$
S_{\text {safe }}\left(v_{p}, v_{f}, a_{\max }, \delta\right)=\frac{1}{2 a_{\max }}\left(v_{f}^{2}-v_{p}^{2}\right)+v_{f} \delta
$$

where $v_{f}, v_{p}$ are respectively the velocity of the following and preceding vehicles, $a_{\max }$ is an acceleration potential and $\delta$ is the reaction delay. We take into consideration the difference of reaction time between a human being and a machine with $\delta_{\text {human }}=2 s$ [10] and contrary to human reaction time, there is no work that investigates the average reaction time of automated vehicles, to the best of our knowledge. We will assume $\delta_{\text {machine }}=0.3 \mathrm{~s}$ from experience with autonomous vehicles, see [6].

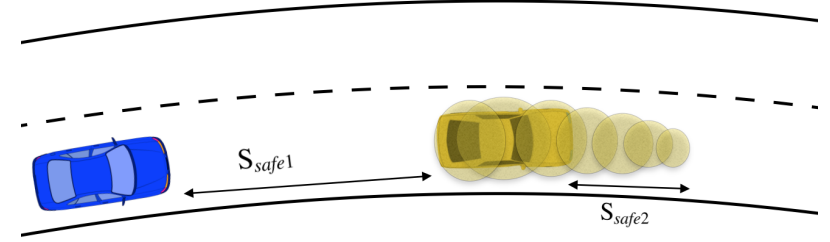

Figure 3: The obstacle shadow after expansion.

The expansion is made by adding circles with varying radius. $S_{\text {safe } 1}$ (Fig. 3) represents the safety distance to keep before overtaking, this distance is taken into account during the trajectory planning part (see III). $S_{s a f e 2}$ (Fig. 3) is the second safety distance to keep between the two vehicles in the end of the overtaking maneuver.

The occupancy grid is modified in order to take into account the presence of a moving obstacle (Fig. 2- $c$ ). After applying the mask to the PerceptionGrid, we look for obstacles in the road. An obstacle is considered present if a certain number of occupied cells is detected. Then, a longitudinal extending of the obstacle is done by changing the masses attributed to the front cells within a safety distance. The spatial propagation modeling of an obstacle according to its velocity is inspired by the discounting operation method. The proposed method increases $m(O)$ of the cells in front of the obstacle. A factor $\alpha$ is specified, and the operation is defined as following:

$$
\begin{gathered}
{ }^{\alpha} m(O)=(1-\alpha) \cdot m(O)+\alpha \\
{ }^{\alpha} m(\mathrm{~B})=(1-\alpha) \cdot m(\mathrm{~B}) \quad \forall B \subseteq \Omega, B \neq O
\end{gathered}
$$

With $m(O)$ is the mass on Occupied state. The decay factor $\alpha$ decreases in the same way as the radius of the circles used for expansion.

\section{THE TRAJECTORY PLANNING AND THE REWARD SYSTEM}

\section{A. Clothoid tentacles for trajectory planning}

At a local on-road level, the trajectory planning goal is the computation of an obstacle free route while following a desired global reference trajectory defined on a global map. The generated trajectory must satisfy the vehicle's kinematic limits based on vehicle dynamics and constrained by the navigation comfort, respect lane boundaries and traffic rules, while avoiding, at the same time, static and dynamic obstacles.

One used technique for a local on-road trajectory planning is based on a search space which contains a specific geometric curve (e.g. clothoids or splines) with several lateral shifts of this curve [11],[9]. Each generated curve is then evaluated with regard to certain criteria.

In this work, we use the clothoid tentacles method. This method is based on generating a set of clothoids tentacles as possible trajectories on an egocentric occupancy grid around the vehicle [7]. The main advantage of the clothoid approach is taking into consideration the current dynamical state of the vehicle and making smooth variation in the vehicle dynamic variables. 
For a fixed velocity, all tentacles begin at the center of gravity of the vehicle and take the shape of clothoid (Fig. 4).

We assume that all tentacles generated for a given speed $V_{x}$ have the same length:

$$
L_{\text {tentacle }}(m)=\left\{\begin{array}{cc}
t_{0} V_{x}-L_{0} & V_{x}>1(\mathrm{~m} / \mathrm{s}) \\
2(m) & V_{x} \leq 1(\mathrm{~m} / \mathrm{s})
\end{array}\right.
$$

where $t_{0}=7 \mathrm{~s}$ and $L_{0}=5 \mathrm{~m}$.

The initial curvature $\rho_{0}$ of the tentacles is calculated from the current vehicle steering angle $\delta_{0}$.

$$
\rho_{0}=\frac{\tan \delta_{0}}{L}
$$

where $L$ is the vehicle's wheelbase.

Tentacles of the extremity correspond respectively to the positive and negative maximal value of the reached steering angle which the vehicle can make at the current velocity without losing stability. The length of tentacles increases with the increase of the velocity.

We assume that all tentacles generated for a given velocity have the same length.

After generating all tentacles in the egocentred occupancy grid related to the vehicle, the next step is to choose the best tentacle to execute using different criteria.

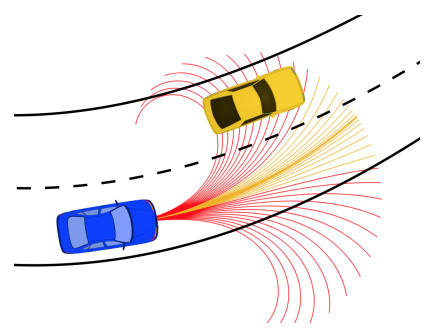

Figure 4: Clothoid tentacles generated at the center of gravity of the vehicle. Red tentacles are non navigable and the yellow ones are navigable.

\section{B. Reward system for choosing the best tentacle}

After generating a set of tentacles, only one must be chosen to be executed. First, a classification is made on the tentacles. They are classified as navigable or non navigable using the information of the occupancy grid. If a tentacle passes by an obstacle on a radius of $S_{s a f e 1}$ in front of the ego-vehicle, this tentacle will be classified as non-navigable, otherwise it's navigable. If a tentacle passes by unknown cells, it's still considered navigable.

After classifying the tentacles, we evaluate the navigable tentacles using several criteria: the tentacle's occupation, its distance from the global reference trajectory and the overtaking criterion.

To model the problem of planning with all the criteria to be taken into consideration, we used a Markov Decision Process (MDP) like model [15]. A $M D P$ is a discrete-time state-transition system. The agent (ego-vehicle) observes the state (environment around each tentacle) and performs an action (tentacle execution) accordingly.
The system then makes a transition to the next state and the agent receives some reward.

It can be described formally with 5 components $(S, A, T, R, \gamma): S$ is the set of states represented here by circles around the tentacles , $A(s): S \rightarrow A$ is the set of actions (each tentacles represents an action), $T: S \times S \times A \rightarrow[0,1]$ defines the transition probabilities of the system from one state to another when taking an action, $R: S \times A \rightarrow \mathbb{R}$ is the reward given to each state dependung on different criteria and $\gamma \subset[0,1)$ is the discount rate used to calculate the long-term attenuation.

\section{Reference trajectory criterion}

The reference trajectory is the path that the ego-vehicle must follow all the time. However, the ego-vehicle can deviate temporarily from the reference trajectory to avoid obstacles or to carry out an overtaking. We use the lateral distance between each tentacle and the reference trajectory to evaluate the tentacles regarding this criterion. Details are presented in [15].

\section{Overtaking criterion}

In the case of the presence of an obstacle in front of the vehicle, the tentacles of the left receive a small additional reward since the overtaking is done by the left.

\section{Occupancy criterion}

Each tentacle is evaluated in regard of its occupation. Grid information is used to assign appropriate rewards for each tentacle.

A tentacle discretization is made by using circles (their diameter represent the width of the vehicle with a margin of security) (Fig. 5). This discretization helps us to judge the tentacle's occupancy and serves to have a support zone around the tentacle which will allow the ego-vehicle to circulate in a secure manner. Rewards are given to each circle based on its occupancy.

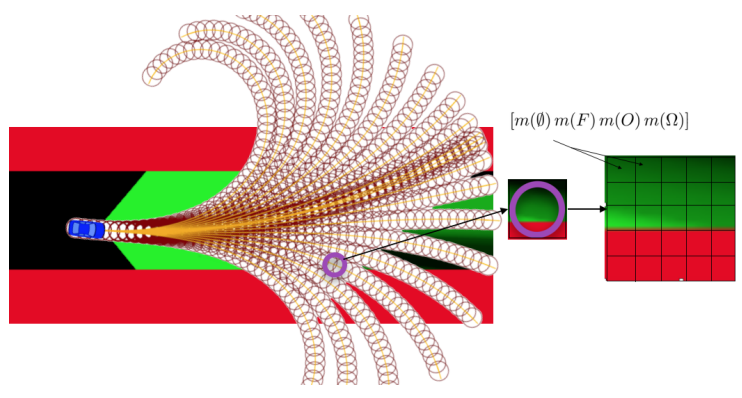

Figure 5: Each tentacle is discretized using circles

For trajectory and overtaking criteria, we used the same reward as presented in [14]. Then the tentacle reward is:

$$
\begin{gathered}
R(\text { tentacle })=\sum_{k=0}^{n_{s}} \gamma_{t}^{k} R\left(s_{k \mid \text { trajectory }}\right)+\sum_{k=0}^{n_{s}} \gamma_{o}^{k} R\left(s_{k \mid \text { occupancy }}\right) \\
+R(\text { overtaking })
\end{gathered}
$$

where $\gamma_{t}$ and $\gamma_{o}$ (Equ. 6) are discount factors that can be used to change the behavior of our approach, and that represent distance attenuation of each kind of reward. $n_{s}$ is the number of state per tentacle, $s_{k}$ is the state number $k$ in the tentacle. 
For occupancy criterion, we used in our previous works binary grid with the value ' 0 ' for free cells and '1' for occupied cells. With evidential grids, instead of having the value '0' or '1' in the grid cells, we dispose of mass about each cell occupancy. Explanations on how we integrate occupancy reward will be provided in the next section.

\section{Reward definition based on evidential grid}

We dispose of an evidential grid in which we draw states as circles around each tentacle. The superposition of the states on the grid gives matrix storing belief mass values (Fig. 5).

In order to define a reward regarding the occupancy of the state, we propose to process cells information using four different rules. We consider that each cell is a source of information about the occupancy of the state. All cells are defined in the same frame of discernment. For each rule, we attribute a different reward (Equations 6 to 9, where $a_{1}, a_{2}, a_{3}, a_{4}$ are weighting parameters):

- Conjunctive rule: the first rule consists on combining all masses of the state matrix with conjunctive rule, the resulting mass function is $m_{\cap}()=\cap m_{i}() \quad \forall$ cell $_{i} \in$ matrix.

$$
\begin{gathered}
\text { Reward }_{\text {occupation }}=a_{1} m_{\cap}(\mathrm{F})+a_{2} m_{\cap}(O) \\
+a_{3} m_{\cap}(\Omega)+a_{4} m_{\cap}(\phi)
\end{gathered}
$$

The conjunctive rule is used if all sources of information are telling the truth. By applying this rule, we obtain a consensus between all sources of information.

- Dempster's rule: the second rule combines all masses of the state matrix with Dempster's rule, the resulting mass function is $m_{\oplus}()=\oplus m_{i}() \forall$ cell $_{i} \in$ matrix.

$$
\text { Reward }_{\text {occupation }}=a_{1} m_{\oplus}(F)+a_{2} m_{\oplus}(O)+a_{3} m_{\oplus}(\Omega)
$$

The normalization process in Dempster's rule has the effect of distributing the belief of conflict to the other propositions, according to their respective mass.

- Mean of the masses: The third combination is a mean of all masses for each state matrix $m_{\text {mean }}()=$ $\operatorname{mean}\left(m_{i}()\right) \forall$ cell $_{i} \in$ matrix.

$$
\begin{aligned}
\text { Reward }_{\text {occupation }}= & a_{1} m_{\text {mean }}(F)+a_{2} m_{\text {mean }}(O) \\
& +a_{3} m_{\text {mean }}(\Omega)
\end{aligned}
$$

- Cells number: with this rule, we count the number of occupied, free and uncertain cells of the state matrix by making a decision about their state. For that, we attribute the element $A \in 2^{\Omega}$ if $m(A)>0.5$.

$$
\text { Reward }_{\text {occupation }}=a_{1} N b(F)+a_{2} N b(O)+a_{3} N b(\Omega)
$$

\section{EXPERIMENTAL AND SIMULATION RESULTS}

\section{A. System set-up and real example}

There are three sources in our perception system: vehicle pose, exteroceptive acquisition data and a map. First, a globally referenced pose is needed to localize the vehicle in the environment in terms of position and orientation compared with reference trajectory. The pose is provided by a GPS system coupled with an inertial measurement unit. Secondly, we use a Lidar as a perception sensor. This sensor can distinguish between free and occupied space and model it in $2 D$ ( $x, y$ coordinates) with respect to the vehicle body frame. We assume that we have the velocity of obstacles. In the validation tests, we used two vehicles one with the Lidar sensor and the second one served as an obstacle to overtake with the velocity information. Finally, the map data with information about the road surface are used.

The Lidar data was acquired at $10 \mathrm{~Hz}$ frequency. The egomotion between two acquisitions is estimated using $C A N$ data. For the purpose of demonstration, the scan grids of (20 * 40) meters are built with uniform cells of size $(0.1 * 0.1)$ meters. We used the evidential grids generated by a $\mathrm{C}++$ code [13] with data acquired on the experimental platform PACPUS in Heudiasyc Laboratory. Fig. 6 shows the road's map where the test is performed.

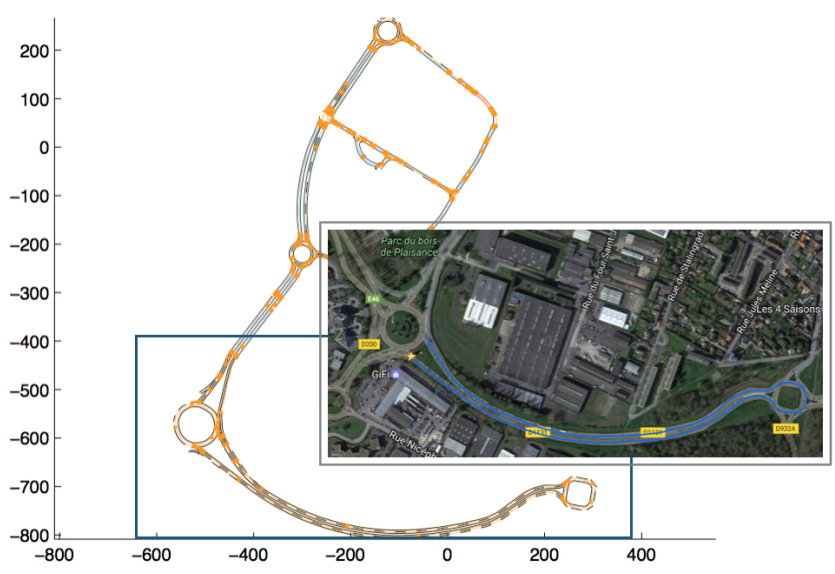

Figure 6: The road's map where the test is performed

One typical scene is chosen to compare the different combination approaches. The resulting PlanGrid is shown in Fig. 7. The ego-vehicle velocity was set at $20 \mathrm{~m} / \mathrm{s}$, and the preceding vehicle's velocity was $14 \mathrm{~m} / \mathrm{s}$.

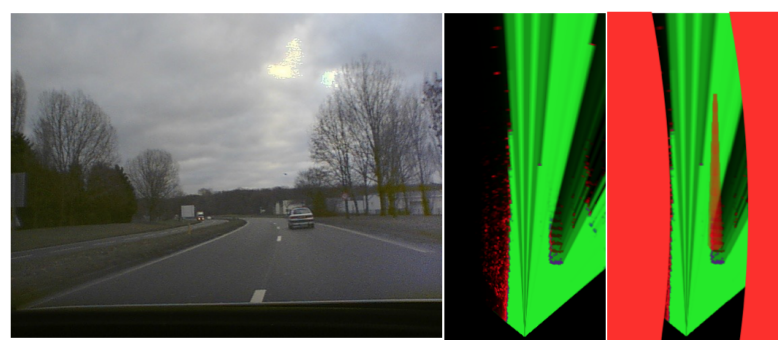

(a)

(b)

(c)

Figure 7: (a) represents a picture of the scene. (b) represents corresponding evidential occupancy grid. $(c)$ represents the expansion of the mobile obstacle with road's edges adding.

Several values of the parameters of the combination rules were tested to find the right values to adopt. 


\begin{tabular}{|c|c|c|c|c|}
\hline Rule & $a_{1}: 1 \rightarrow 100$ & $a_{2}:-100 \rightarrow-1$ & $a_{3}:-20 \rightarrow 10$ & $a_{4}$ \\
\hline \hline Conj. & 10 & -10 & -1 & -10 \\
\hline Demp. & 50 & -20 & -1 & - \\
\hline Mean & 10 & -50 & -1 & - \\
\hline Cell-N. & 20 & -50 & -2 & - \\
\hline
\end{tabular}

Table I: Parameters of different combination rules

\section{B. Results with real data}

During our tests, we collected perception data as evidential grids. These grids have been processed with Matlab as an input to our planning algorithm. We tested the different rules of combination in an overtaking situation using the PlanGrid of Fig. 7. The criterion used to compare them is the safety distance at the end of the overtaking maneuver and the calculation time.

And in order to compare with the binary grids, we transform the PerceptionGrid (Fig. 7-b) into a binary grid using pignistic transformation. A cell is considered to be occupied if $\operatorname{bet} P(O)>\operatorname{bet} P(F)$, and free otherwise; with $\operatorname{bet} P(O)=$ $m(O)+\frac{1}{2} m(\Omega)$ and $\operatorname{bet} P(F)=m(F)+\frac{1}{2} m(\Omega)$.
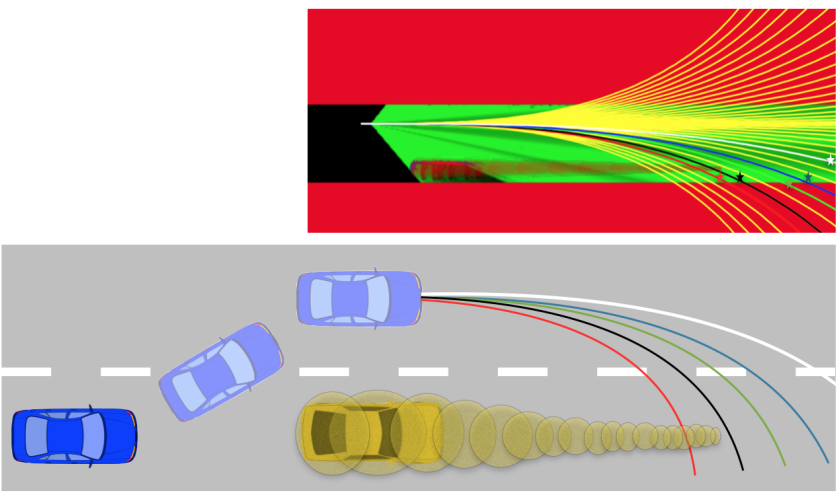

Figure 8: The chosen tentacle among a set of 41 tentacles with four different combinations rules. Mean rule: red, Cell-N rule: black, Conjunctive rule: green and Dempster rule: blue. In white, the chosen trajectory with binary grid.

\begin{tabular}{|c|c|c|c|c|c|}
\hline Rule & Conjunctive & Dempster & Mean & Cell-number & B.Grid \\
\hline \hline Time $(s)$ & $3,3 T$ & $4,7 T$ & $1,3 T$ & $T$ & $0.7 T$ \\
\hline
\end{tabular}

Table II: Time computing for different combination rules and for the binary Grid. The $T$ represents the duration of an iteration.

The result shows that the use of evidential grid enables us to process information about the unknown differently from the occupied space. In Fig. 8, we observe that the binary grid choice is more conservative than the evidential grids. For example with the meand rule combination, the algorithm has chosen the red tentacle which passes through the borders of the obstacle ; On these borders, the mass on the occupied is less important than the mass on a real obstacle. And since the algorithm is attracted by the reference trajectory, its choice is a compromise between safety distance and the return to the reference trajectory. However, the used combination rules require a significant computation time (Table. II).

\section{Simulation Results}

To validate the algorithm on more challenging scenarios, we use SCANeR ${ }^{\mathrm{TM}}$ Studio simulator to get data for simulation. The data was processed in Matlab. From this simulator data, we created a global map with a reference trajectory. We positioned one static obstacle and one dynamic obstacle with a constant velocity of $10 \mathrm{~m} / \mathrm{s}$ (Fig. 9). The ego-vehicle is the blue car (Fig.9). The distance between the static and dynamic obstacle is chosen to include the safety distance that the ego-vehicle need to respect after overtaking manoeuver with a margin of security. We consider that the driver of the yellow car is a human with $\delta_{\text {human }}=2 s$ and $a_{\max }=-5 \mathrm{~m} / \mathrm{s}^{2}$. Using Eq. 3, $S_{\text {safe } 2}=12.5 \mathrm{~m}$.

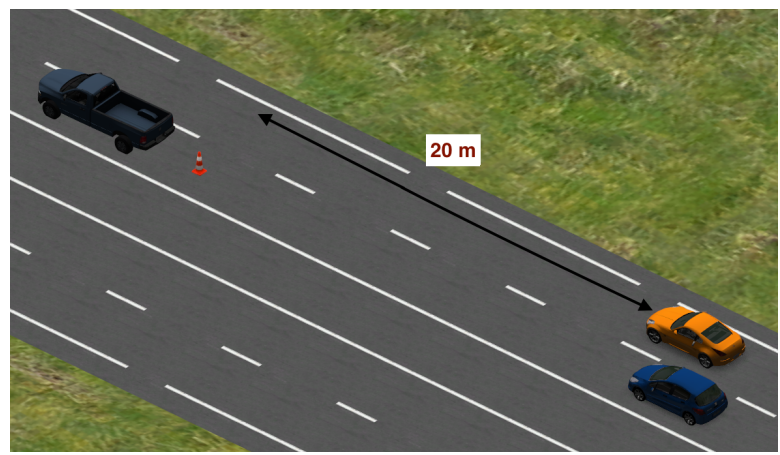

Figure 9: Scenario test from SCANeR ${ }^{\mathrm{TM}}$ Studio simulator. The blue vehicle is the ego-vehicle with $15 \mathrm{~m} / \mathrm{s}$ velocity, the yellow vehicle is a dynamic obstacle with $10 \mathrm{~m} / \mathrm{s}$ velocity and the pick up is the static obstacle.

In order to show the interest of using evidential grids instead of binary grids, we played this scenario with both types of grids.

To construct the binary grid, road borders were considered to be occupied as well as the two dynamic and static obstacles. With obstacle expansion using binary grids, no tentacles are navigable. The ego-vehicle brakes and stops.

To simulate an evidential grid, we affect 4 masses for each grid cell. If the cell with the coordinate $(i, j)$ is on the navigable space, $m[i, j]=[m(\emptyset) m(F) m(O) m(\Omega)]$, where $m(F)=\operatorname{rand}(0.5,1)$ (random number between 0.5 and 1), $m(O)=0, m(\Omega)=1-m(F)$ and $m(\emptyset)=0$. If the cell is on the occupied space; $m(O)=\operatorname{rand}(0.5,1)$, $m(F)=0, m(\Omega)=1-m(O)$ and $m(\emptyset)=0$. The right and left borders of the road and behind obstacles in the evidential grid represent a lot of uncertainties. In order to represent this uncertainty, if the cell is in the road borders or behind obstacles; $m(\Omega)=\operatorname{rand}(0.5,1), m(F)=1-m(\Omega)$, $m(O)=0$ and $m(\emptyset)=0$. In every time step, we update the grid.

With obstacle expansion using evidential grids, the algorithm can find navigable tentacles thanks to the spatial propagation modeling of an obstacle using discount masses.

Simulation results in Fig. 10 show that the use of the evidential grids makes it possible to differentiate between occupied and uncertain space. Thus, the overtaking maneuver is carried out despite the uncertain situation. The binary grid 


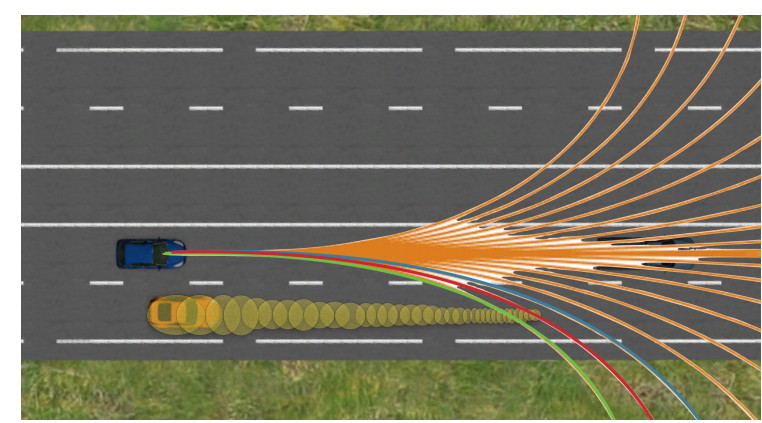

Figure 10: The chosen trajectory with four different combinations rules. Mean rule, Cell-N: green, Conjunctive rule: red and Dempster rule: blue.

does not allow to find a solution while respecting the safety distance (the ego vehicle brakes and stops) whereas with the evidential grids, we improve robustness to uncertainty of some road's situation.

\section{Conclusion And Perspectives}

In this work, the goal is to manage uncertainties of the environment for trajectory planning using evidential grids. Therefore, the potential use of the evidential grids was tested in different ways during the reward process to select the best tentacle. Real and simulation data were used and processed with Matlab to evaluate the performances of these approaches. The first results show that the use of evidential grids improves our planning algorithm robustness to uncertainty of some road's situation. As perspective, we look for more experiments in order to choose the best combination rule and show the potential of this approach specially in urban autonomous driving. However, the used combination rules require a significant computation time which makes its use in real time challenging. The next experiments will allow us to make the compromise between efficiency and computation time.

\section{ACKNOWLEDGMENT}

This work was carried out in the framework of the the Equipex ROBOTEX (Reference ANR-10-EQPX-44-01) and the Labex MS2T (Reference ANR-11-IDEX-0004-02) which were funded by the French Government, through the program "Investments for the future" managed by the National Agency for Research.

\section{REFERENCES}

[1] M. Althoff and R. Losch. Can automated road vehicles harmonize with traffic flow while guaranteeing a safe distance?. IEEE International Conference on Intelligent Transportation Systems Conference (ITSC), pp. 485-491, 2016.

[2] J. M. Alvarez, T. Gevers, Y. LeCun, and A. M. Lopez. Road scene segmentation from a single image. In European Conference on Computer Vision, pp. 376-389, pages 376-389, 2012.

[3] S. Brechtel, T. Gindele, and R. Dillmann. Probabilistic decision-making under uncertainty for autonomous driving using continuous POMDPs. Intelligent Transportation Systems (ITSC), 2014 IEEE 17th International Conference on, 28, 2014.

[4] A.P. Dempster. Upper and lower probabilities induced by a multivmulti mapping. The Annals of Mathematical Statistics, 38, 1976.
[5] C. Fulgenzi, A. Spalanzani, and C. Laugier. Dynamic obstacle avoidance in uncertain environment combining pvos and occupancy grid. IEEE International Conference on Robotics and Automation, pp. 1610-1616., 2007.

[6] M. Goebl, M. Althoff, M. Buss, G. Farber, F. Hecker, B. HeiBing, S. Kraus, R. Nagel, F.P. Leon, F. Rattei, and M. Russ. Design and capabilities of the munich cognitive automobile. In Intelligent Vehicles Symposium, pp. 1101-1107, 2008.

[7] M. Himmelsbach, T. Luettel, F. Hecker, V. Hundelshausen, and H.-J. Wuensche. Autonomous off-road navigation for mucar-3, improving the tentacles approach: Integral structures for sensing and motion. Kunstl Intell, 2011.

[8] M.L. Littman, A. R. Cassandra, and L. P. Kaelbling. Learning policies for partially observable environments: Scaling up. Proceedings of the Twelfth International Conference on Machine Learning Machine Learning Proceedings 1995, p. 362, 1995.

[9] L. Ma, J. Yang, and M. Zhang. A two-level path planning method for on-road autonomous driving. In: 2012 2nd International Conference on Intelligent System Design and Engineering Application, pp. 661-664, 2012.

[10] D.V. McGehee, E.N. Mazzae, and G.S. Baldwin. Driver reaction time in crash avoidance research: Validation of a driving simulator study on a test track. In Proceedings of the human factors and ergonomics society annual meeting, 44(20), pp.3-320, 2000.

[11] M. Montemerlo, J. Becker, S. Bhat, D. Dolgov, H. Dahlkamp, S. Ettinger, D. Haehnel, T. Hilden, G. Hoffmann, B. Huhnke, D. Johnston, D. Langer, S. Klumpp, J. Levinson, A. Levandowski, J. Marcil, D. Orenstein, J. Paefgen, I. Penny, and A. Petrovskaya. Junior : The Stanford Entry in the Urban Challenge. Journal of Field Robotics, 25(9), pp. 569-597, 2008.

[12] J. Moras, V. Cherfaoui, and P. Bonnifait. Credibilist occupancy grids for vehicle perception in dynamic environments. IEEE International Conference In Robotics and Automation (ICRA), pp. 84-89, 2011.

[13] J. Moras, V. Cherfaoui, and P. Bonnifait. Moving objects detection by conflict analysis in evidential grids. IEEE Intelligent Vehicles Symposium (IV), pages 1122 - 1127, 2011.

[14] H. Mouhagir, R. Talj, V. Cherfaoui, F. Aioun, and F. Guillemard. Integrating safety distances with trajectory planning by modifying the occupancy grid for autonomous vehicle navigation. IEEE International Conference on Intelligent Transportation Systems Conference (ITSC), pp. 1114-1119, 2016.

[15] H. Mouhagir, R. Talj, V. Cherfaoui, F. Aioun, and F. Guillemard. A markov decision process-based approach for trajectory planning with clothoid tentacles. International IEEE Conference on Intelligent Vehicles Symposium (IV), pp. 1254-1259, 2016.

[16] G. Shafer. A mathematical theory of evidence. Princeton University Press, 1976.

[17] P. Smets and R. Kennes. The transferable belief model. Artificial Intelligence, 66, pp. 191-234, 1994.

[18] $\mathrm{Ph}$. Smets. Decision making in the tbm: the necessity of the pignistic transformation. Int.ernational Journal of Approximate Reasoning, 38, pp.133-147, 2005.

[19] J. Wei, J. M. Dolan, J. M. Snider, and B. Litkouhi. A point-based mdp for robust single-lane autonomous driving behavior under uncertainties. IEEE International Conference on Robotics and Automation (ICRA), pp. 2586-2592., 2011.

[20] C. Yu, V. Cherfaoui, and P. Bonnifait. Semantic evidential lane grids with prior maps for autonomous navigation. IEEE 19th International Conference on Intelligent Transportation Systems (ITSC), pp. 1875 1881,2016 